\title{
On limitations of studies and limitations of therapy options for carotid stenosis: why play golf with only a sand wedge?
}

\author{
Jens Fiehler
}

Received: 13 April 2010 /Accepted: 13 April 2010 /Published online: 14 May 2010

(C) Springer-Verlag 2010

I am grateful for having the chance to edit this issue of Neuroradiology focused on intra- and extracranial stenting directly after and in response to the publication of ICSS [1] and presentation of CREST [2] - two major randomised controlled trials (RCTs) comparing carotid endarterectomy (CEA) and carotid artery stenting (CAS). The results and more so, the interpretation of these studies may have a substantial impact on the neuroradiological community. Based on this data pooled with other RCTs, it has been advocated that "routine use of CAS in patients with recent symptoms of carotid stenosis who are suitable for endarterectomy can no longer be justified" [3]. This carefully worded statement leaves some room for interpretation, particularly in regard to what "suitable for endarterectomy" means. In effect, some reader might go so far as to interpret this conclusion as general call to abandon CAS as a method. Many neuroradiologists feel that this is not a good option as their local experiences with CAS are much better than the results that have emerged from the trials. An example is reported in a single-centre experience within this issue [4]. However, is such data useless since operators tend to be favourable in assessing their outcome [5]? Are results of observational studies necessarily overruled by the publication of RCTs? I do not think so. Observational studies can outweigh the disadvantages of RCTs in many situations [6]. RCTs may suffer some own inherent problems in design and conduct that limit their general-

J. Fiehler $(\bowtie)$

Department of Neuroradiology, Universitätsklinikum Eppendorf,

Martinistrasse 52,

20246 Hamburg, Germany

e-mail: fiehler@uke.uni-hamburg.de isability, and often these flaws are detectable only after the trial is complete [7].

Evidence-based medicine has overcome the traditional paradigm of medical practise, which was solely based on intuition, pathophysiological rationale and unsystematic clinical experience. This traditional model is certainly insufficient grounds for clinical decision making in our time. However, we must not replace this old scheme by uncritical and direct translation of study data to therapy decisions. Personally, I feel uneasy by about naïve positivism disguised as decision making on scientific grounds.

This issue will start with a critical review of the European-led trials that have been published so far (EVA3S, SPACE, ICSS) [8]. This result of a discussion among neuroradiologists from a variety of European countries is not intended to be a political argument against the concept of evidence-based medicine; instead, it follows the fundamentally important concept of scientific work: criticism. We need to reflect on the application of evidencebased medicine to Interventional Neuroradiology permanently. If we do not do this, others will. If we do not engage with evidence-based medicine, we increase the risk of RCTs that largely neglect procedural standardisations and operator expertise. Such studies might have higher enrolment rates, but they do no not necessarily produce useful "evidence". The resulting data is a guaranteed playground for simplification and misinterpretation. Beware of surgical trials organised by nonsurgeons [9]. It is up to us to get involved into the discussion even more actively. By no means should we regress to the age of "comic opera" that we considered to have left behind with ISAT [10].

Instead of becoming frustrated and stepping backwards into the presumed heroic past with individual 
decisions by single experts, we need to rethink our methods of scientific investigation in order to better prepare for future RCTs. First, we need to improve the quality of our case series according to international standards [11]. Second, more imaging information should be used as covariate (e.g., morphology of carotid bifurcation and the plaque). Comparing real outcomes with multivariate predicted ones based on imaging information might help in selecting suitable patients and estimating effect sizes for later RCTs $[12,13]$. Third, we need to investigate the mode of learning of interventional procedures and the influence of the operator (as surgeons have done in their field for years already). Fourth, intelligent methods of database research and data mining could be employed [14].

Given the low incidence and the anatomical heterogeneity of several diseases we treat in Interventional Neuroradiology, there are considerable difficulties in reaching sufficient patient numbers for RCTs (e.g., AVMs, dAVFs). Other fundamental problems for the conduct of RCTs in Interventional Neuroradiology include continuous and sometimes rapid evolution of technology (rendering older results useless), lack of blinding (placebo operations are rarely agreed to), missing patient consent (in case of grossly differing study interventions) and missing consent of interventionalist (no clinical equipoise presumed), among others. For example, with strict criteria for defining precluding problems in an ideal clinical research setting, less than $40 \%$ clinical treatment evaluation questions in surgery could be answered by a RCT [15].

The relatively high incidence and high anatomical uniformity of carotid stenosis and the possibility of therapy standardisation renders it one of the best suitable diseases for investigation in our discipline. The low incidence of intracranial stenosis in European and North American population might be one of the reasons why intracranial stenting is done without information from a RCT so far. The scientific basis is discussed in a review paper within this issue [16]. Several stents are used in clinical practise: some are approved for intracranial application and some are approved for extracranial use only (such as coronary stents). A large case series on such stent [17] will be discussed in a guest editorial on the practise and implications of off-label use in Europe. In contrast, there are plenty of commercially available dedicated carotid stents (with room for improvement in stent design). Hopefully, designing new stents and other trials will improve future patient care. The present challenge after publication of the CEA vs. CAS RCTs is to integrate both methods into a joint infrastructure so that each patient receives optimal care. A neurorologist's view [18] demonstrates what such structure, based on the most recent data, could look like. For instance, in patients up to and including 68 years of age, risk of a primary outcome event seems lower after CAS than after CEA; whereas, the risk was lower for older patients treated by CEA than for those treated by CAS in two large and independent RCTs [2, 19]. Such analyses generate hypotheses, and we need to proceed with secondary pooled analyses and with trials that incorporate improved design and patient selection based on lessons learned from previous trials.

Limiting the therapy options in carotid stenosis patients based on the current data is a serious consideration. Premature rejection of CAS could harm future patients that would have had a benefit from this procedure. It is hard to believe that only a single therapy option is optimal for every carotid stenosis patient we see even if the pooled RCT results show superiority of CEA over CAS in periprocedural stroke risk. This is like throwing away all your golf clubs and keeping only your sand wedge because it showed the best average performance in the hands of the average golf player. Would a golf professional expect winning the European Open with only a sand wedge?

Conflict of interest statement I declare that I have no conflict of interest in the field of carotid stenting. I have previously received speaker's fees from Boston Scientific, ev3 and Cordis Neurovascular.

\section{References}

1. Ederle J, Dobson J, Featherstone RL et al (2010) Carotid artery stenting compared with endarterectomy in patients with symptomatic carotid stenosis (International Carotid Stenting Study): an interim analysis of a randomised controlled trial. Lancet 375:985997

2. Brott $\mathrm{T}$; Investigators $\mathrm{ftC}$ (2010) The randomized Carotid Revascularization Endarterectomy vs Stenting Trial (CREST): primary results. In: International Stroke Conference. San Antonio, TX, USA: Stroke, Abstract 197

3. Rothwell PM (2009) Poor outcomes after endovascular treatment of symptomatic carotid stenosis: time for a moratorium. Lancet Neurol 8:871-873

4. Tietke M, Kerby T, Alfke K et al (2010) Complication rate in unprotected carotid artery stenting with closed cell stents. Neuroradiology, in press

5. Rothwell $P$, Warlow $C$ (1995) Is self-audit reliable? Lancet 346:1623

6. Shrier I, Boivin J, Steele R et al (2007) Should meta-analyses of interventions include observational studies in addition to randomized controlled trials? A critical examination of underlying principles. Am J Epidemiol 166:1203-1209

7. Sackett D, Hoey J (2000) Why randomized controlled trials fail but needn't: a new series is launched. CMAJ 162:1301-1302

8. Fiehler J, Bakke SC, Clifton A et al (2010) Plea of the defencecritical comments on the interpretation of EVA3S, SPACE and ICSS. Neuroradiology, in press

9. Kassell N, Dumont A (2006) Randomized controlled trials in surgery: comic opera no more? J Thorac Cardiovasc Surg 132:243-244 
10. Sellar R, Whittle I (2004) The lessons learnt from ISAT: surgical research is rescued from comic opera. Br J Neurosurg 18:405-406

11. von Elm E, Altman D, Egger M, Pocock S, Gøtzsche P, Vandenbroucke J (2007) The Strengthening the Reporting of Observational Studies in Epidemiology (STROBE) statement: guidelines for reporting observational studies. Ann Intern Med 147:573-577

12. Wu O, Christensen $\mathrm{S}$, Hjort $\mathrm{N}$ et al (2006) Characterizing physiological heterogeneity of infarction risk in acute human ischaemic stroke using MRI. Brain 129:2384-2393

13. Ries T, Wulff A, Säring D, Wegscheider K, Fiehler J (2010) Unexpected kink in aneurysm recurrence volume distribution is associated with retreatment decision. Neuroradiology, in press

14. Schaap M, Metz C, van Walsum T et al (2009) Standardized evaluation methodology and reference database for evaluating coronary artery centerline extraction algorithms. Med Image Anal $13: 701-714$
15. Solomon M, Mcleod R (2005) Should we be performing more randomized controlled trials evaluating surgical operations? Surgery 118:459-467

16. Kurre W, Chapot R, du Mesnil de Rochemont R, Berkefeld J (2010) Intracranial stenting in atherosclerotic disease-recent results and challenges to face. Neuroradiology, in press

17. Vajda Z, Miloslavski E, Güthe T, et al. (2010) Treatment of intracranial atherosclerotic arterial stenoses with a balloonexpandable Cobalt Chromium Stent (Coroflex Blue): procedural safety, efficacy and mid-term patency. Neuroradiology, in press

18. Rosenkranz M (2010) Diagnostic workup in carotid stenosis - a neurologist's perspective. Neuroradiology, in press

19. Ringleb PA, Allenberg J, Bruckmann H et al (2006) 30 day results from the SPACE trial of stent-protected angioplasty versus carotid endarterectomy in symptomatic patients: a randomised noninferiority trial. Lancet 368:1239-1247 